\title{
A Novel Protocol to Provide Authentication and Privacy in WSN
}

\author{
Devi.T, N.Deepa, K.Lakshmi Swathi, Harthik, Manideep
}

\begin{abstract}
A wireless sensor network holds a large amount of nodes. These nodes will contact themselves by utilizing some of the radio signals. wireless sensor networks (WSNs) has develop some applications during a huge selection areas, in the time of which external side users ought to straightly attach with sensors to get a perceived information. But, WSNs (wireless sensor node) are open to numerous attacks for wireless links, like eavesdropping and meddling. Two-factor authentication combining password and ID utterly like this demand due to password and ID usefulness. Then, a bucket of two-factor authentication protocol was advised in present research works. Because of the difficult assignment of adjustable potency and privacy requirements, still it's difficult to introduce a privacyaware two-factor protocol that's potential of giving different safety features whereas take care of proper potency. in this paper the proposed work tend to suggests a privacy aware two-factor authentication protocol depend on ECC for wireless sensor nodes(WSNs). In this another convention performs distinctive wellbeing highlights need fully for the application situations, all things considered, though deal with appropriate power. So in this we will in general demonstrate that the presented convention accomplishes intelligent in the Burrows-Abadi-Needham judgment to boot, through manner of unofficial security statistics, the work show the introduced protocol will face up to a range of attacks and supply fascinating safety features.
\end{abstract}

Keywords: Elliptic curve cryptography, Gateway node, Sensor nodes, Two factor authentication.

\section{INTRODUCTION}

In today world privacy plays a crucial role in every life. We have to secure all the things by using various passwords. For that Wireless sensor networks (WSNs) shall be utilized to fulfill real-time observance in different environments. Networked sensors will simply be stationed in different environments. Usually, the gateway node consists of high power \& capability, whereas the wireless sensors have unsufficient, memory, CPU power and storage capability, computational capability. Usually normally a person called user must interact to the sensors in order to obtain the detected information.

Revised Manuscript Received on J(uly 22, 2019.

Devi.T, Assistant Professor, Saveetha School of Engineering, SIMATS, Chennai.

N.Deepa, UG Student, Saveetha School of Engineering, SIMATS, Chennai.

K.Lakshmi Swathi, UG Student, Saveetha School of Engineering, SIMATS, Chennai.

Harthik, UG Student, Saveetha School of Engineering, SIMATS, Chennai.

Manideep, UG Student, Saveetha School of Engineering, SIMATS, Chennai.

By taking all resources of sensors, the authentication protocol of a user for wireless sensor nodes (WSNs) must be economical like calculation price. Therefore, the capability utilization of the decreased crypto-graphical algorithms must be decreased during pointing the safety needs. For solving the problem of planning a private two factor security protocol, one of the privacy-aware two-factor protocol can be pointed by varied issues of security with some of the resource sensors and detected information was designed.

Let the stiffness of plotting a private two-factor security protocol, for privacy-aware two-factor protocol its tough that come together on varied private needs with relevancy there source constraints of sensors.

\section{LITERATURE REVIEW}

In 2009, Firstly Das used two-factor authentication combining password as well as ID for determining authentication problems with wireless sensor nodes (WSNs) that causes user security for wireless sensor nodes (WSNs) to a new way. Then, it had been visible that the security protocol of that author cannot guarantee correlative authentication, or user non recognition and is unprotected to several attacks, session key negotiation as well as normal offline password approximation attack, GWN bypassing attack, denial-of-service (DOS) attack, sensor node capture attack, and three upgraded security protocols for wireless sensor networks (WSNs) were arranged to control these problems.

Then, Yoo et al. exhibit some older security protocols quiet be wretched from various security attacks as well as limitations and planned an upgraded protocol to abolish these marks. The proposed system acknowledged some of the older protocols quiet have few security disadvantages within a lot of sensible condition that some of the confidential parameters hold on in ID are often removed once it is gone. Therefore, they recommend an upgraded protocol continues to be private even once the ID is gone. Kumar et al. additionally acknowledged the protection marks in one of the theme. So therefore the theme of the that author as well as suggests one strong two-factor security theme, giving correlative security, and password update, session key agreement.

A while later, another system proposed goes with two disservice concerning security and assurance. It is unsuccessful to achieve un-perceptibility and encounters taken ID strike. In this way, they have given an extended affirmation show to fix these two obstructions. Sun et al. described that the theme of system still encounters various ambushes concerning the GWN. 


\section{A Novel Protocol to Provide Authentication and Privacy in WSN}

To take out these disservices, they moreover propose a made show. In any case, it's anything but difficult to watch out that the show of system still doesn't take customer security affirmation into thought as well as achieves neither regular check between the customer and thusly the GWN nor key comprehension among customers as well as sensors. The system is incontestable that theme of another system in addition dubious against various strikes as well as it's not moderate inside part of essentialness usage for WSNs. to manage these security vulnerabilities as well as restrictions, they proposed a staggering approval show with security confirmation. Other than customer check shows maintained symmetrical key philosophy, collection of elliptic twist cryptography (ECC)- based affirmation shows are masterminded fails to supply customer mystery expression change instrument and is vulnerable against insider attack. The system masterminded an ECC-based two-factor approval subject. In any case, in the new ECC-based point, the customer and discoverer can't similarly demonstrate one another. To fix these issues, Shi et al. organized an improved ECC-based check subject. Differentiated and the arrangement of the point gives a lot of security incorporates and performs higher to the extent figuring and correspondence. Be that as it may, a system discovered that the confirmation subject of Shi et al. is in risk of cloud key offer ambush, purloined splendid card attack, and pointer essentialness draining strike. To abstain from these security deficiencies, they conjointly orchestrated an improved affirmation show. Sadly, the show of work still can't accomplish anonymity and un-perceptibility. Around a similar time, the system was given an ECC-based approval theme that gives un-detect ability.

\section{PROPOSED WORK}

Firstly, in registration phase users registers itself with gateway node $(\mathrm{GWN})$, users selects the person ID and password and erratically selects a number. Next the person totalize it and sends to gateway node (GWN). By obtaining the request, gateway node (GWN) enquires validity of ID as well as reject the request of ID does not confirm requirements of user's identity. Then GWN calculates the temporal credential of user and sensor node and protected temporal credential of user and then stored. Finally GWN issues the ID which consists of hash function, current timestamp and protected temporal credential to user.

Then, in login phase user need to access sensor node. User insert the ID into terminal as well as enters ID as well as password. Smart card calculates the password of user. If password does not take the card reject the request Otherwise calculates the temporal credential of sensor node. In authentication phase communicating agents commonly authenticate every other also gives the session key (Fig.1).

In changing password phase a user have to modify the password and inserts the person card into workstation and give the ID \& password. The ID calculates the password of user. If password doesn't take the card reject the request. Otherwise users remove the old password and create a new password and calculate protected temporal credential.

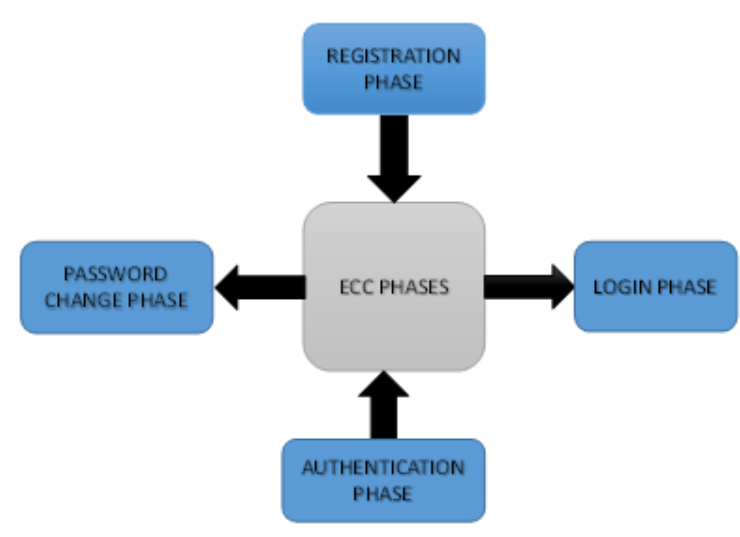

Fig. 1 Proposed System Phases

\section{RESULTS AND DISCUSSION}

ECC algorithm uses a 160 bit key size which is very less compared RSA that makes use of 1024 keys. As the key size decrease the time for encryption and decryption also decreases. In addition to this, the security provided also increases which proves the efficacy of the system (Fig.2).

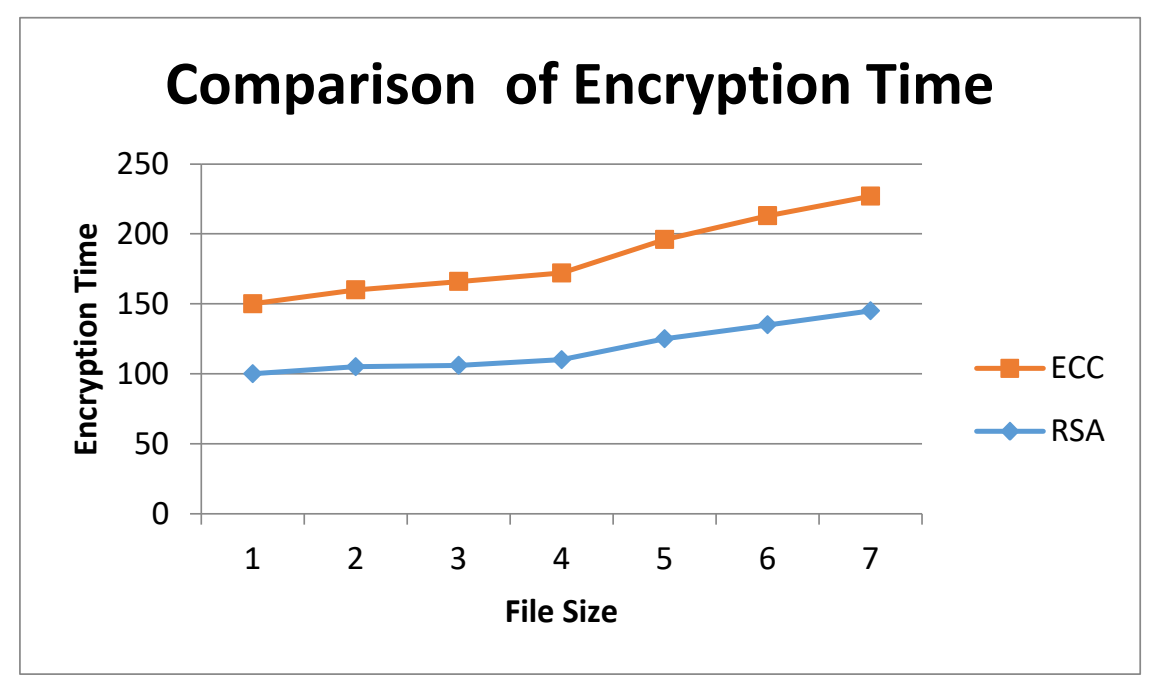

Fig. 2 Encryption using ECC 


\section{CONCLUSION}

The existing system introduced a privacy-aware twofactor security or authentication protocol mistreatment error correction code for wireless sensor network (WSNs). That author suggest that their protocol accomplish different privacy and usefulness options needful for present applications whereas keep up allowable potency. Therefore, this work examined Jiang protocol and exhibited that protocol has some security openness, like a scarcity of different security, a risk of such modification attacks, unavailability sensor obscurity, and faint ID obscurity. For resolve these openness, a privacy-upgrade privacy-aware two-factor authentication protocol exploitation error correction code for WSNs has to be projected.

\section{REFERENCES}

1. Xie S, Wang Y. Construction of tree network with limited delivery latency in homogeneous wireless sensor networks. Wireless Personal Communications 2014; 78(1): 231-246.

2. Shen J, Tan H, Wang J, Wang J, Lee S. A novel routing protocol providing good transmission reliability in underwater sensor networks. Journal of Internet Technology 2015; 16(1): 171-178.

3. He D, Zeadally S. Authentication protocol for an ambient assisted living system. Commun. Mag. IEEE 2015; 53(1): 71-77. 4. He D, Zeadally $\mathrm{S}, \mathrm{Wu} \mathrm{L}$. Certificateless public auditing scheme for cloudassisted wireless body area networks. IEEE Systems Journal 2015. DOI:10.1109/JSYST.2015.2428620.

4. Ren Y, Shen J, Wang J, Han J, Lee S. Mutual verifiable provable data auditing in public cloud storage. Journal of Internet Technology 2015; 16(2): 317-323.

5. Ren Y, Shen J, Zheng Y, Wang J, Chao H-C. Efficient data integrity auditing for storage security in mobile health cloud. Peer-to-Peer Networking and Applications 2015. DOI:10.1007/s12083-015-0346-y.

6. Guo P, Wang J, Li B, Lee S. A variable threshold-value authentication architecture for wireless mesh networks. Journal of Internet Technology 2014; 15(6): 929-936.

7. He D, Zeadally S, Kumar N, Lee J. Anonymous authentication for wireless body area networks with provable security. IEEE Systems Journal 2016. DOI:10.1109/JSYST.2016.2544805.

8. He D, Kumar N, Shen H, Lee J. One-to-many authentication for access control in mobile pay-TV systems. Science China Information Sciences 2016. DOI:10.1007/s11432-015-5469-5, 2016.

9. Wang D, He D, Wang P, Chu C-H. Anonymous two-factor authentication in distributed systems: Certain goals are beyond attainment. IEEE Transactions on Dependable and Secure Computing 2015; 12(4): 428-442. DOI:10.1109/ TDSC.2014.2355850.

10. Wang D, Wang N, Wang P, Qing S. Preserving privacy for free: Efficient and provably secure two-factor authentication scheme with user anonymity. Information Sciences 2015; 321: 162-178. DOI:10.1016/j.ins.2015.03.070.

11. Xia Z, Wang X, Sun X, Wang Q. A secure and dynamic multi-keyword ranked search scheme over encrypted cloud data. IEEE Transactions on Parallel and Distributed Systems 2015 DOI:10.1109/TPDS.2015.2401003.

12. Fu Z, Sun X, Liu Q, Zhou L, Shu J. Achieving efficient cloud search services: Multi-keyword ranked search over encrypted cloud data supporting parallel computing. IEICE Transactions on Communications 2015; E98-B(1): 190-200.

13. Li H, Yang Y, Luan T, Liang X, Zhou L, Shen X. Enabling finegrained multi-keyword search supporting classified sub-dictionaries over encrypted cloud data. IEEE Transactions on Dependable and Secure Computing 2015. DOI:10.1109/TDSC.2015.2406704. 